\title{
Once bitten, twice shy: An exploratory study of victim impact from and adoption of self-protective behaviours against cyber abuse
}

\author{
Zarina I. Vakhitova*, Rob I. Mawby ${ }^{\dagger}$ Clair L. Alston-Knox ${ }^{\ddagger}$
}

September 6, 2019

\begin{abstract}
Background and purpose of the research: The impact of crime on its victims is reflected in the expansion of victim services across the world since the 1970s. These policy developments have largely expanded alongside research on how crime affects victims. Most established research has focused on conventional crimes such as burglary, sexual and violent offences, with little of the published research examining the impact of online crime like cyber abuse. The current paper explores a relatively new subject, the impact of cyber-abuse on its victims and the factors associated with the adoption of self-protective behaviours following victimization from different types of cyber abuse.

Methodology: To better understand the impact of cyber abuse on its victims, the data from a large nationwide (US) crowd-sourced sample $(N=1,463)$ of adult members of an online labour portal Mechanical Turk was collected using an online survey platform Qualtrics. Around half of the respondents reported experiencing at least one type of cyber abuse victimization $(N=746)$ and were included in the sample analyzed in this study. To identify the factors predictive of higher victim
\end{abstract}

*zarina.vakhitova@monash.edu

†ProfessorRobMawby@hotmail.com

${ }^{\ddagger}$ calston-knox@pa-group.com.au 
impact and adoption of self-protective behaviours, the data was modelled using a Bayesian variable selection procedure implemented via a stochastic search algorithm in AutoStat@.

Results: Our findings suggest that first, being a female and having a prior relationship with the abuser is associated with an increased victim impact from cyber abuse victimization. The type of abuse experienced by the victim has not been found to be predictive of the level of impact. Second, the adoption of self-protective behaviours was significantly associated with a higher perceived victim impact, older victim, and the mixed type of cyber abuse.

Conclusion/discussion: Findings from this study contribute to both our understanding of cyber abuse as a broad crime category, the mechanism of adoption self-protective behaviours following victimization, as well as help inform policy responses to the needs of cyber abuse victims.

Keywords: cyber abuse, victimization, impact of crime, self-protective behaviours, Bayesian variable selection, stochastic search algorithm.

\section{Introduction}

Crime is a significant social issue that affects millions of people around the world every year. In the US, in 2017, 3 in every 1,000 Americans experienced violence and 24 in every 1,000 Americans were victims of property crime (Gramlich, 2019). With the advent of the Internet and other telecommunication technologies over the past several decades, novel methods of committing crimes have been introduced. According to Gallup's annual crime survey, as many as one in four U.S. households experienced cybercrime victimization in 2018 (Reinhart, 2018) ${ }^{1}$.

Crime can negatively affect many areas of victims' lives, including their physical health, employment or education prospects, finances and emotional well-being (Dinisman and Moroz, 2017; Fisher et al., 2000; Shapland and Hall, 2007; Tjaden and Thoennes, 2000). The extent of the impact of crime on its victims varies:

\footnotetext{
${ }^{1}$ In fact, cybercrime victimization now is much more common than burglary, assault and robbery experienced by only about 14,2 , and $1 \%$ of American households respectively.
} 
Experiencing a crime can affect people in many ways, and every person's reaction is different. At the start, you might feel shocked, fearful or angry. A common reaction is feeling numb, and not believing that this has happened to you. You might suffer from: headaches, nausea, sleep problems or fatigue, jumpiness, repeated thoughts of the event. Or you might have feelings that come and go, like: guilt, fear, anger, sadness, confusion, helplessness. Your day-to-day life might be affected by: trouble with your concentration or memory, reduced performance at work or school, withdrawing from others, feeling like you've lost control. Later, some people can experience depression or anxiety (Victim Support Agency, n.d.).

Besides the aforementioned psychosomatic reactions to crime, a common response to victimization is the adoption of self-protective behaviours. These actions could take the form of modifying the usual daily routines, undertaking protective actions such as carrying a weapon, changing the locks and installing an alarm system in the house, withdrawing from social activities, moving, changing the contact information (e.g. email address), reporting victimization to the police or undertaking some judicial action such as obtaining a restraining order against the offender, seeking an assistance from friends, family, co-workers or others, and other similar behaviours (Baum et al., 2009; Fisher et al., 2002; Tjaden, 1998; Buhi et al., 2009; Reyns et al., 2011; Sheridan and Grant, 2007; Wilcox et al., 2007). Like with psychosomatic reaction to crime, the adoption of self-protective behaviours is not uniform (Nobles et al., 2014), and, as a result, the factors that determine an individual's reaction to criminal victimization is not yet clear.

The existing research suggests that the impact of crime and the subsequent reaction vary according to the offence type and victim characteristics. The brunt of research about victim impact so far focused on conventional crimes like burglary and assault. However, with the proliferation of new types of technology-facilitated crimes such as cyber abuse, it became necessary to explore the mechanisms of victimization and the victim's reaction to victimization in the context of these new types of crime. With this in mind, the present study addresses two issues related to the impact of cyber abuse 
victimization on its victims, which are currently largely unexplored. First, we identify the factors predictive of victim impact from different types of cyber abuse. Second, we identify the determinants of adopting self-protective behaviours following cyber abuse victimization.

This study contributes to both the knowledge base on cyber abuse victimization, its mechanisms and impact on victims, as well as the literature on adopting self-protective behaviours following incidents of personal victimization. In practical terms, this research has a potential not only to assist in improving crime prevention strategies, especially for preventing repeat victimization but also to ensure that victims receive the help they need.

\section{The effect of offence type on victim impact}

Victims of serious interpersonal attacks, such as sexual and other violent offences tend to be severely affected by crime with long-term consequences (Holmstrom and Burgess, 1978). Research commissioned by the Victim Support England and Wales, UK, noted:

Victims of violence describe feelings of shock and loss of trust in society, and guilt at becoming a victim of crime, as they typically feel they could have prevented the incident from occurring. Violent crime can also cause victims to feel a sense of uncertainty and dis-empowerment and to feel more vulnerable, leading to high levels of worry about personal safety. Violent victimization has also been found to be linked to the development of symptoms of fear, anxiety, depression or confusion, sadness, anger and stress (Dinisman and Moroz, 2017, p. 4).

On the other hand, property crimes are generally considered less harmful to victims $^{2}$. However, studies based on the analyses of Crime Survey England and Wales and British Crime Survey data suggested that burglary victims may also be severely affected.

\footnotetext{
${ }^{2}$ As reflected in the development of various crime harm indexes around the world, see for example, Cambridge Crime Harm Index (Sherman et al., 2016)).
} 
In contrast to victims of violent crimes, victims of vehicle-related crimes tend to react in the form of anger rather than fear or anxiety (Mawby, 2001; Mawby and Walklate, 1997; Shapland and Hall, 2007). Summarizing the findings, Mawby and Walklate (1994) concluded that victims were most affected when the crime was serious, interpersonal, and was likely to occur again rather than be a 'one-off'.

While most established research has focused on conventional crimes such as burglary, sexual and violent offences, more recently the effects of online crime such as cyber abuse $^{3}$ have been studied (Sheridan and Grant, 2007; Hensler-McGinnis, 2008; Dreßing et al., 2014; Nobles et al., 2014; Short et al., 2014; Short et al., 2015; Worsley et al., 2016; Fissel, 2018; Fissel and Reyns, 2019). Research suggests that, much like the victims of conventional crimes, victims of cybercrimes such as cyber abuse experience a variety of negative effects including persistent psychological impairment and issues of personal insecurities (Parsons-Pollard and Moriarty, 2009; Dreßing et al., 2014; Nobles et al., 2014).

Sheridan and Grant (2007) found that both offline and online stalking had a serious impact on victims, with no significant differences in terms of medical and psychological consequences and only minor differences in social and financial consequences between the two types of abuse. In contrast, Nobles et al. (2014) found some significant differences between online and offline stalking with victims of cyber stalking less likely to report fear of crime but higher out-of-pocket costs associated with the incident of victimization. Victims of cyberstalking were also more likely to report adopting self-protective behaviours compared with victims of offline stalking. Short et al. (2015) compared the victim impact from offline and online harassment and found that both could result in high levels of psychological distress comparable to the effects of post-traumatic stress disorder. In a different setting, Hensler-McGinnis (2008) examined a sample of university students and found that cyberstalking victimization was significantly associated with trauma and impairment in academic/career functioning. Fissel and Reyns (2019) distin-

\footnotetext{
${ }^{3}$ Cyber abuse is a wide crime category that includes any behaviour that aims to stalk or harass an individual over the age of 18 using technology. In this paper, we review studies that examined cyber abuse, cyberstalking and cyberharassment as these terms significantly overlap and often used interchangeably.
} 
guished between four categories of consequences of cyberstalking victimization - health, social, work and school. In a sample of victims aged 18 to 25 , the researchers found that when online stalking was combined with offline stalking the risk of experiencing one or more of types of consequences of victimization was much higher.

Dreßing et al. (2014) examined the impact of cyberstalking in a sample of members of the German social network. The researchers found that the victims commonly experienced some form of negative psychological or health-related outcome, including a general feeling of inner unrest, mistrust towards other people, sleep disorders, feeling of helplessness, anger, aggression, and a variety of health problems including headaches, depression, and panic attacks. Similar, Short et al. (2014), who conducted an in-depth analysis of cyberstalking victim narratives, found that victims experienced psychological, physical and social consequences as a result of victimization. To summarize, the research suggests that cyber abuse can affect its victims in different ways and, in general, consequences to the victim can be quite serious.

\section{The effect of victim characteristics on impact from crime}

While it is clear that the impact of crime varies for different subgroups of victims, the actual mechanism of variation, including the predictive factors, as well as whether these mechanisms are common between different categories of crime is less obvious. In an offline context, an earlier study by Mawby and Walklate (1994), using British Crime Survey data, reported that the most vulnerable victims were most affected: poorer people living in rented accommodation, nonwhites, divorced victims and those living in single adult households; older citizens, and women. Stalking research identified prior offendervictim relationship, and gender of the victim as consistent predictors of higher victim impact (Baum et al., 2009; Davis et al., 2002; Korkodeilou, 2017). Sheridan and Lyndon (2012), in an online survey of self-defined stalking victims, found that females were more likely than males to experience more severe psychological and physical consequences 
of stalking victimization compared to males, as were those who had had a close prior relationship with the perpetrator. Johnson and Kercher (2009) analysis of the Texas Crime Victimization Survey also identified women, those with a prior relationship with the stalker, and those who were divorced or separated as particularly affected.

Cyber abuse research that focused on predictors of victim impact is currently limited to only two studies. Dreßing et al. (2014) found that prior intimate partner relationship between the offender was a factor in cyberstalking victimization. Unsurprisingly, the researchers found the majority of offenders to be males and conversely, the majority of victims to be females. Fissel and Reyns (2019) distinguished the impact of cyberstalking on victims on four dimensions - work, school, social and health and concluded that incidents that lasted longer, included more online pursuit behaviours, and also involved offline stalking victimization had a greater impact (Fissel and Reyns, 2019). However, in contrast to research on offline stalking their findings on the relationship between gender and offender-victim relationship and impact were ambiguous. Both studies treated cyber abuse as one uniform crime. To date, no study that we know of examined victim impact from different forms of cyber abuse victimization. A better understanding

of mechanisms of victim impact from different types of cyber abuse is needed to ensure the appropriate level and type of victim support.

\section{Predictors of adoption of self-protective behaviours as a reaction to victimization}

One way, not yet discussed here, in which the effect of victimization can be expressed and observed most readily, is in the adoption of self-protective behaviours following victimization. Research shows that victims of conventional types of crime such as stalking adopt a variety of different self-protective behaviours including changing their daily routines, moving homes, or undertaking prevention measures in the form of carrying a weapon (Baum et al., 2009). The research in identifying predictors of adopting self-protective behaviours in the context of conventional crimes such as stalking is quite limited. Earlier 
work by Gottfredson and Hindelang (1979) found that identified seriousness of the crime as important in the victim's decision to report the crime to the police in the National Crime Survey (US).

Cho et al. (2019) focused on predictors of help-seeking behaviour and the use of formal and informal sources of help (e.g. medical or legal help) by the victims of intimate partner violence (IPV). This research is of particular interest to this study as cyber abuse often occurs in the context of $\mathrm{IP}^{4}$. The researchers found that victim's characteristics, patterns of victimization and consequences of victimization determined help-seeking behaviours, while gender moderated the relationship between consequences and the use of formal help.

The literature on adopting self-protective behaviours following stalking victimization is relatively well developed (Baum et al., 2009; Buhi et al., 2009; Fisher et al., 2002; Reyns et al., 2011; Sheridan and Grant, 2007; Tjaden, 1998; Wilcox et al., 2007). Victims of stalking often report adopting avoidant behaviours, moving to a different location, undertaking security protective measures (e.g. installing alarms, security cameras, changing locks, etc.), taking preventive actions (e.g. carry pepper spray), seeking assistance from third parties or pursuing judicial or disciplinary action responses (e.g. reporting to the police) (Baum et al., 2009; Nobles et al., 2014; Tjaden, 1998). In the context of cyber abuse, content analysis of cyber abuse victimization survey conducted by Tokunaga and Aune (2017) identified seven self-protection techniques used by victims including ignore/avoidance, active technological disassociation, help seeking, negotiation/threat, compliance/excuses, technological privacy management and derogation, with using technology to move away from pursuers reported as the most effective self-protection tactic.

Due to the diverse nature of self-protective behaviours, researchers operationalized self-protective behaviours using a variety of different measures. In the context of cyber abuse victimization, Fissel (2018) operationalized self-protective behaviours as re-

\footnotetext{
${ }^{4} \mathrm{~A}$ report by Ybarra et al. (n.d.) estimates that as many as $12 \%$ of U.S. internet users have experienced intimate partner digital abuse. The same report identified that $77 \%$ of victims of intimate partner digital abuse have used at least one protective strategy; one in six have gotten a restraining order or protection order as a result of their digital abuse experiences.
} 
porting incidents of victimization to the police. Worsley et al. (2016), who focused on the psychological impact of cyberstalking, conceptualized self-protective behaviours as behaviours aiming to cope with the stress associated with experiencing victimization and was measured as avoidant coping, ignoring the perpetrator, confrontational coping, support seeking and cognitive re-framing. Sheridan and Grant (2007), who compared victims impact and response to victimization between stalking and cyberstalking victims measured self-protective behaviours using the following indicators: changing employment, changing the course of study, changing/getting rid of the car, undertaking some security measures, going underground/changing identify, and giving up social activities.

In terms of specific predictors of adopting self-protective behaviours by victims of cyber abuse, the literature, as Nobles et al. (2014, p. 993) suggested, "has not developed to the point where patterns in responses to victimization, including self-protective behaviours taken by the victim, have been clearly identified". The one study in this area is by Nobles et al. (2014) who examined the factors that influence of the adoption of self-protective behaviours among victims of cyber stalking. The study focused on the seriousness of the experienced incident of victimization operationalized through the presence of physical attacks, threats, financial costs to the victim, duration of stalking, level of experienced fear, and acknowledgement of experience as stalking. The researchers aimed to predict the number of self-protective behaviours adopted by cyberstalking victims using the seriousness of the event and controlling for several demographic characteristics (Nobles et al., 2014). Nobles and colleagues found that almost all variables measuring the seriousness of victimization included in the model were significant predictors of adopting self-protective behaviours. Specifically, the results indicated that victims who experienced greater out-of-pocket costs, greater fear at the onset, and those who considered their experiences to be stalking engaged in significantly more self-protective behaviours ${ }^{5}$. No study, that we are aware of, has examined the nature of the relationship between the perceived impact of cyber abuse victimization and the adoption of self-protective behaviours against different types of cyber abuse victimization. This gap in knowledge is

\footnotetext{
${ }^{5}$ These findings should be treated with caution however as the sample of cyberstalking victims analyzed in this study was quite small $(N=296)$.
} 
significant and addressing it will allow researchers and practitioners not only to better understand the mechanisms of adopting self-protective behaviours but also to develop more targeted prevention strategies against cyber abuse.

In summary, this literature review suggests that while research on the impact of crime on victims is extensive, the expansion of internet crime has brought a new dimension to victimization and victims' experiences, and little of the published research relates directly to this. The current paper thus explores a relatively new subject, the impact of cyber-abuse on its victims.

\section{The current study}

The impact of cyber abuse can be addressed on at least four levels. First, given that victims of conventional crime report being affected in various ways, we can ask whether cyber abuse victims are similarly affected. Second, we can address the question of how they are affected: in psychological terms or through changing behaviour patterns (in particular, the ways they use technology). Third, we can consider variations among cyber abuse victims according to victim characteristics: that is, are different subgroups of victims (e.g. distinguished by age) affected more than, or in different ways to others. Fourth, we can consider variations according to the subcategories of cyber abuse discussed above: that is, are those subjected to a particular type of cyber abuse (e.g. direct) affected more than, or in different ways to, others, just as different types of crime trigger different victim response and have a different perceived impact?

In this study, we follow Ellison and Akdeniz (1998) classification of cyber abuse, which groups different related behaviours according to the method used to perpetrate them as either direct or indirect cyber abuse. In this context, direct cyber abuse is delivered by the offender directly to the victim via abusive emails or text messages, by posting comments on the victim's social media pages, or through covert surveillance of the victim's activities (Vakhitova et al., 2018). In contrast, indirect cyber abuse is delivered with minimal or no direct interaction between the victim and the offender. Indirect cyber abuse does not require knowledge of the victim's personal contact information. 
These methods of abuse include posting damaging information about the victim on a non-victim's social media pages or web sites (Vakhitova et al., 2018). This updated classification of cyber abuse is particularly useful if we consider the potential to develop targeted crime prevention strategies. It would appear logical that behaviours that involve direct contact between offenders and victims would require a prevention approach different from the one needed to prevent behaviours that do not require direct contact.

With this in mind, the current study draws from two areas of research - cyber abuse and lifestyles and routine activities as predictors of victimization to identify factors associated with 1) victim impact from cyber abuse and 2) adoption of self-protective behaviours following cyber abuse victimization. In this study, we focus on one type of self-protective behaviours - changes in the way victims use technology to reflect the inherently technological nature of cyber abuse.

To answer our research questions we model the mechanisms of victimization using the indicators of victims' lifestyles and routine activities as well as their demographic characteristics. Findings from this study contribute to both understanding of cyber abuse as a broad crime category as well as help inform policy responses to the needs of cyber abuse victims. This study contributes to our understanding of new technology-facilitated types of crime such as cyber abuse by identifying the determinants of individual reaction to cyber abuse victimization and, specifically, of adopting self-protective behaviours following cyber abuse victimization.

\section{Methodology}

The research reported here used an online survey instrument. Cyber abuse, due to its technological nature, is especially suited for exploring using an online survey. Online surveys are also associated with reduced interviewer-induced measurement error (Baker et al., 2010; Sue and Ritter, 2012) and social desirability bias (Chang and Krosnick, 2009; Kreuter et al., 2008) when compared to face-to-face and telephone interviews.

The survey was designed to collect the information about the experience of our respondents with cyber abuse victimization, the effect it had on their lives and their re- 
lationship with technology, and demographic characteristics. All of the survey questions were original, developed especially for this study. It took our respondents om average no longer that 15 minutes to complete the survey.

A non-probability (convenience) sample drawn from the members of Amazon's Mechanical Turk, an online contract labour portal, formed the foundation for data analysis in this study. As the focus of this study was on cyber abuse, the phenomenon, which by definition involves adults only, only those 18 years of age or older US residents were invited to participate in the survey. Crowd-sourced samples, like the one we've collected, allow for access to a large and fairly diverse pool of potential respondents (Behrend et al., 2011; Berinsky et al., 2012; Buhrmester et al., 2011).

We limited participation to workers who were at least 18-years-old and who resided in the United States. The workers were offered a small monetary compensation (US 35c) for their participation in the research. This amount was chosen based on the minim hourly wage in the USA and the estimated average amount of time required to complete a survey. Research suggests that this approach improves response quality in Mechanical Turk surveys (Peer et al., 2013).

The rate of missing data in the survey — both from break-off and item non-responsewas low. In total, 1,623 workers began the survey, and 1,463, or slightly over $90 \%$, completed the survey. To identify victims of cyber abuse among those who accepted our invitation to participate in the survey, we first asked all our respondents whether they experienced any form of cyber stalking or cyber harassment directed at them personally. To ensure that the respondents were clear about the types of behaviours we were interested in, we provided a number of examples of different forms that cyberstalking and cyber abuse ${ }^{6}$. Out of the total number of respondents, around half $(N=746)$ reported experiencing some form of cyber abuse. Only responses from those individuals were included in the final sample analyzed herein.

\footnotetext{
${ }^{6}$ Here are the examples of cyber abuse behaviours we provided our respondents: 1) emails, texts (SMS), posts on blogs, online forums and social media pages of a persistent, annoying, alarming or threatening nature, 2) monitoring your daily activities using social media or specialized software, 3) posting information about your online (photos, documents, videos) without your consent or distribution such information to others via email, SMS or other technological means, 4) impersonating you online or through email or SMS, 5) subscribing you online to unwanted services, products, activities, etc.
} 


\section{Measures}

\section{Dependent variables}

To answer our research questions, we have constructed two dependent variables: 1) Overall Effect (continuous: $\min =0, \max =2,1$ decimal place) and 2) Adoption of self-protective behaviours (dichotomous: yes $=1$, no $=0$ ). To collect this information we asked our respondents the following two questions:

Question 1: How affected were you by this incident (psychologically, emotionally, financially, or otherwise)?

Question 2: Did this incident cause you to change the way you conduct your online activities and/or use technology? (Yes, No)

To answer the first question, the respondents picked a position on a slider anywhere between 0 and 2 where 0 meant the respondent was not at all affected by the abuse, 1 somewhat affected and 2 - profoundly affected. Note, in relation to the previous research discussed earlier, that we did not include any follow-up questions on the ways in which victims were affected.

Our analyses include three groups of independent variables: 1) types of cyber abuse victimization, 2) lifestyle and routine activities-related factors at the time of the incident, and 3) demographic controls. Table 1 presents descriptive statistics for all independent variables, demographic controls and situational factors.

\section{Independent variables}

To isolate direct, indirect and mixed types of cyber abuse, we asked the respondents who identified as victims to think about the most recent or the most memorable incident they experienced, and to select one or more specific behaviours they experienced from the list: 1) you received a text message, email or a private message via social media addressed to you personally, 2) someone posted derogatory, embarrassing information (documents, photos, videos, etc.) about you on the Internet or distributed to others via email, text 
(SMS) or other technology, 3) you have been subscribed to unwanted services, products, activities. You only found out about the subscription after you started to receive the services, products or invited to participate in the activities, 4) someone, pretending to be you, sent email or text messages or private messages on social media pages to your family, friends, co-workers, or other third parties, 5) your daily activities been monitored by someone via social media or a tracking software, 6) someone created a website or a social media page containing derogatory or embarrassing information about you. We then combined the information from these two questions (about the fact of victimization and the specific type of victimization) and created a 3-level factor variable Method (direct $=0$, indirect $=1$, mixed $=2$ ). The resulting variable had levels in following proportions: direct $(n=309)$, indirect $(n=168)$, mixed $(n=269)$.

Further, to assess the nature of offender-victim relationship prior to the abuse, we asked our respondents who was the person(s) involved in the incident in relation to the respondent and provided the following possible responses: a) close or extended family; b) romantic partner or spouse; c) romantic ex-partner or ex-spouse; d) business or work connection (co-worker, colleague, employer, employee or business associate); e) education connection (college or university professor or student, academic advisor or supervisor, tutor, teacher, coach or trainer); f) friend or acquaintance; g) social media or online gaming connection; h) someone I met through sports, social clubs or committees, volunteer organizations etc.; i) a stranger (someone I definitely do not know); j) I am not sure of the real identity of the person(s) involved, but I suspect, it is someone I know; k) a mixed group of people; l) other. Please specify. We then coded responses as follows: a-h and j- 1 - prior relationship and i - 0 - no prior relationship. Responses k and 1 were coded accordingly and following the same principles.

To control for the effect of demographic characteristics on the perceived level of victim impact, we have included the following demographic variables as controls in both our models: age (in years), gender $($ female $=1)$, race/ethnicity $($ white $=1)$, and employment status (employed $=1$ ). To ensure that we measure the demographic characteristics around the time of the cyber abuse incident, we asked our respondents-victims to give 
us the information in the context of the time of the incident they reported. For example, "Around the time of the incident, what was your age?".

\section{Analytic strategy}

To answer the research questions posed in this study, we first conducted univariate and bivariate analyses for our variables of interest and then modelled victim impact and adoption of self-protective behaviours using a generalized linear model ${ }^{7}$. In deciding on the modelling approach, we took into consideration the exploratory nature of the study; the explicit lack of theoretical guidance on selection of variables to be included in our models; and the benefits of statistical methods of variable selection identified in previous literature (see for example, Raftery (1995), Vakhitova and Alston-Knox (2018), and Vakhitova et al. (2016)). With this in mind, a stochastic search algorithm to determine the most likely models which include variables that can explain the outcomes as well as preserving good estimation performance was deemed most appropriate. AutoStat ${ }^{\circledR}$ software was used to estimate these models ${ }^{8}$.

A Bayesian approach to estimation was used to determine the most probable models in terms of explanatory power. These models were then used to estimate the posterior distributions of the unknown parameter effects. In regression, where there are $k$ potential explanatory variables, the full model likelihood can be specified as

$$
y \mid \beta, X, \sigma^{2} \sim N\left(\beta_{K} X, \sigma^{2} I\right)
$$

where $K=\{0,1,2, \cdots, k\}$ possible regressors ( $K=0$ indicates intercept term).

In a Bayesian setting, the unknown parameters, $\beta$ and $\sigma$ require a prior distribution to be specified in order to estimate their respective posterior distributions. Zellner's G-prior (Zellner, 1986) is convenient as it allows us to introduce information about the parameters "mean" location but does not require the specification of the correlation

\footnotetext{
${ }^{7}$ To reflect the specific nature of our two dependent variables, continuous variable Victim Impact and dichotomous variable Adoption of Self-Protective Behaviours, we employed generalized linear models in the form of linear and binary logistic regressions.

${ }^{8}$ To conduct the analyses in this paper we employed AutoStat, a software package for statistical analysis by Predictive Analytics Group (http://pa-group.com.au/autostat.php).
} 
structure between regressors, which is difficult to achieve. The prior distributions for the parameters $\beta_{K}$ and $\sigma$ for the full model are:

$$
\begin{aligned}
\beta_{K} \mid \sigma^{2}, X & \sim N_{k+1}\left(\tilde{\beta}, g \sigma^{2}\left(X^{T} X\right)^{-1}\right) \\
\sigma^{2} & \propto \sigma^{-2}
\end{aligned}
$$

where $g$ is specified to reflect the certainty of the prior. For example, large values of $g$ increase the variance, hence the prior becomes less certain as $g$ increases. In our analysis, we set $g$ equal to our sample size, hence the prior distribution is akin to the influence of one observation. As a result, the posterior distribution is dominated by the data. The hyper-prior $\tilde{\beta}$ is set at zero.

It is often the case that variables in the full model may exhibit collinearity. As a result, assessing the merit of each variable is difficult, as even the sign of the coefficient may give an erroneous impression of that variable's role in the outcome. Reducing the model using variable selection is a common technique for approaching this issue, but as there are $2^{k}$ competing models (assuming that the intercept is always included), statistical analysis requires a strategy to traverse the space.

In this analysis, we used stochastic search variable selection, where each of the $2^{k}$ models $\left(M_{\gamma}\right)$ is associated with a binary vector $\gamma$ where $\gamma_{i}=1$ when $x_{i}$ is in the model and 0 otherwise. As the model probability, $M_{\gamma}$, is now another parameter to be estimated, we assign the prior as a uniform distribution for all models. The stochastic search algorithm of Marin and Robert, 2007 (p82) is then used to determine the posterior probability of each model.

Under this model, the prior for the coefficients included in model $M_{\gamma}$ (known as the "slab") becomes

$$
\beta_{\gamma} \mid \gamma, \sigma^{2} \sim N_{q_{\gamma}+1}\left(\tilde{\beta}_{\gamma}, g \sigma^{2}\left(X_{\gamma}^{T} X_{\gamma}\right)^{-1}\right)
$$

and the prior distribution for $\sigma^{2}$ is unchanged from the full model. Variables in model $M_{\gamma}$ where $\gamma_{i}=0$ are considered to be on the "spike" which is a point mass at zero, and hence $\beta_{i}=0$ in this model. For more information about the prior and the model 
specification please see Marin and Robert (2007).

\section{Results}

Table 1 presents the descriptive statistics for the sample. Means and standard deviations are provided for continuous variables and percentages of the total sample for dichotomous variables. The final sample is similar to the general population of American adults in terms of gender split, but differs in terms of age and race - it is whiter and younger. This is consistent with prior research using Mechanical Turk samples (Levay et al., 2016; Weinberg et al., 2014; Pickett et al., 2018).

Table 1: Descriptive statistics $\left(N_{\text {victims }}=746\right)$

\begin{tabular}{|c|c|c|c|c|c|}
\hline Variables & $\%(\mathrm{~N})$ & Mean & SD & Min & Max \\
\hline \multicolumn{6}{|l|}{ Dependent variables } \\
\hline Perceived impact & & 1.13 & 0.56 & 0 & 2 \\
\hline Adopting self-protective behaviours & $40(299)$ & & & 0 & 1 \\
\hline \multicolumn{6}{|l|}{ Independent Variables } \\
\hline \multicolumn{6}{|l|}{ Type of abuse } \\
\hline Direct & $41(309)$ & & & & \\
\hline Indirect & $23(168)$ & & & & \\
\hline Mixed & $36(269)$ & & & & \\
\hline Prior offender-victim relationship & $78(562)$ & & & 0 & 1 \\
\hline \multicolumn{6}{|l|}{ Demographic Controls } \\
\hline Age & & 30.90 & 10.83 & $13^{* *}$ & 70 \\
\hline Female & $54(399)$ & & & 0 & 1 \\
\hline White & $70(522)$ & & & 0 & 1 \\
\hline Employed & $80(601)$ & & & 0 & 1 \\
\hline
\end{tabular}

*Abbreviations: SD - standard deviation; min - minimum; max - maximum

$* *$ Age at the time of the incident.

All respondents were at least 18 years of age at the time of the survey.

As Table 1 shows, in our sample, direct abuse is much more common than indirect abuse ( $41 \%$ and $23 \%$ respectively) with the majority of victims reporting having a preexisting relationship with the offender, which is highly consistent with previous literature. In terms of the perceived victim impact, the average level in our sample was 1.13 (out of maximum 2) (see Fig. 1 for a histogram of victim impact), which corresponds to slightly higher than "somewhat affected", with almost half of the sample (40\%) reporting adopting some self-protection behaviours (in the form of changing the way they use technology) following the victimization. 


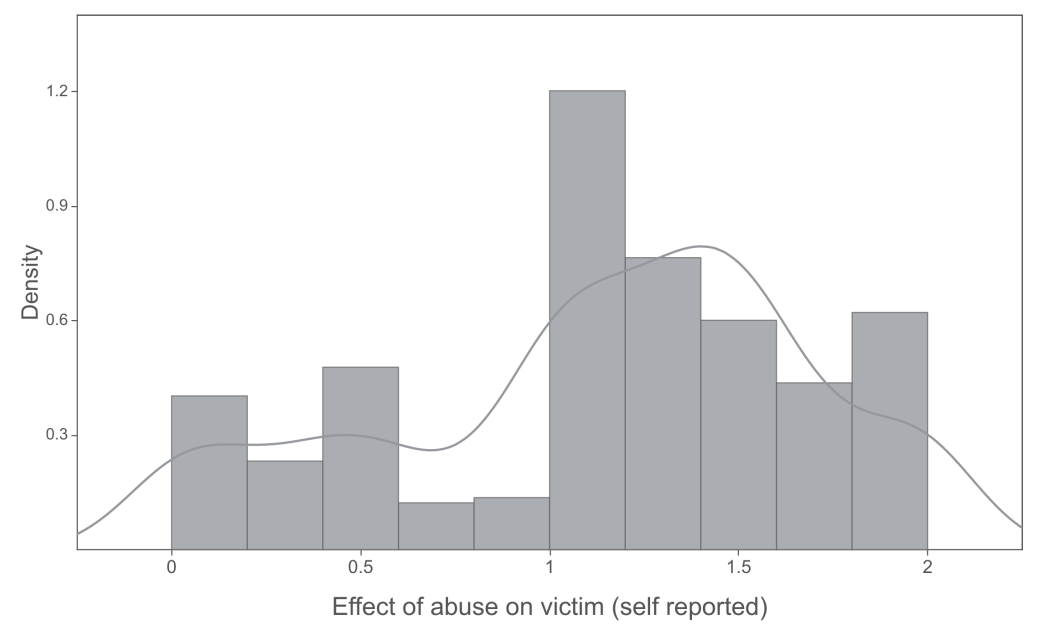

Figure 1: Histogram of victim impact with a density curve

Table 2 presents correlation coefficients for bi-variate relationships for the variables of interest ${ }^{9}$.

Table 2: Correlation matrix for variables of interest

\begin{tabular}{llllllll}
\hline \hline & Variables & 1 & 2 & 3 & 5 & 6 & 7 \\
\hline 1 & Victim impact & & & & & & \\
2 & Adopting self-protective behaviours & $.326^{* *}$ & & & & & \\
3 & Offender-victim relationship & $.146^{* *}$ & $-.100^{* *}$ & & & & \\
4 & Age & -.022 & $.151^{* *}$ & $-.182^{* *}$ & & & \\
5 & Gender & $.132^{* *}$ & $.132^{* *}$ & .007 & $.107^{* *}$ & & \\
6 & Race & -.004 & -.005 & .044 & $.199^{* *}$ & .034 & \\
7 & Employment & .031 & .009 & .034 & .005 & $-.126^{* *}$ & -.040 \\
\hline \hline
\end{tabular}

As Table 2 shows, victim impact is significantly ${ }^{10}$ positively associated with adopting self-protective behaviours, offender-victim relationship and gender ${ }^{11}$, while adopting self-protective behaviours are significantly ${ }^{12}$ positively associated with age and gen-

\footnotetext{
${ }^{9}$ Spearman correlation coefficients were calculated for pairs of continuous variables (dark gray cells), Pearson correlation coefficient for continuous-dichotomous pairs (light gray cells), and Phi coefficient for pairs of dichotomous variables (white cells).

${ }^{10}$ Correlation is significant at the 0.01 level (2-tailed).

${ }^{11}$ Victim impact is also significantly positively associated with employment, but this correlation is only significant at the 0.05 level (2-tailed).

${ }^{12}$ Correlation is significant at the 0.01 level (2-tailed).
} 
der and significantly negatively associated with the offender-victim relationship. The strongest relationship is between the victim impact and adopting self-protective behaviours (see Fig. 2). This association suggests that those victims who experienced higher impact from victimization are more likely to react by adopting self-protective measures.

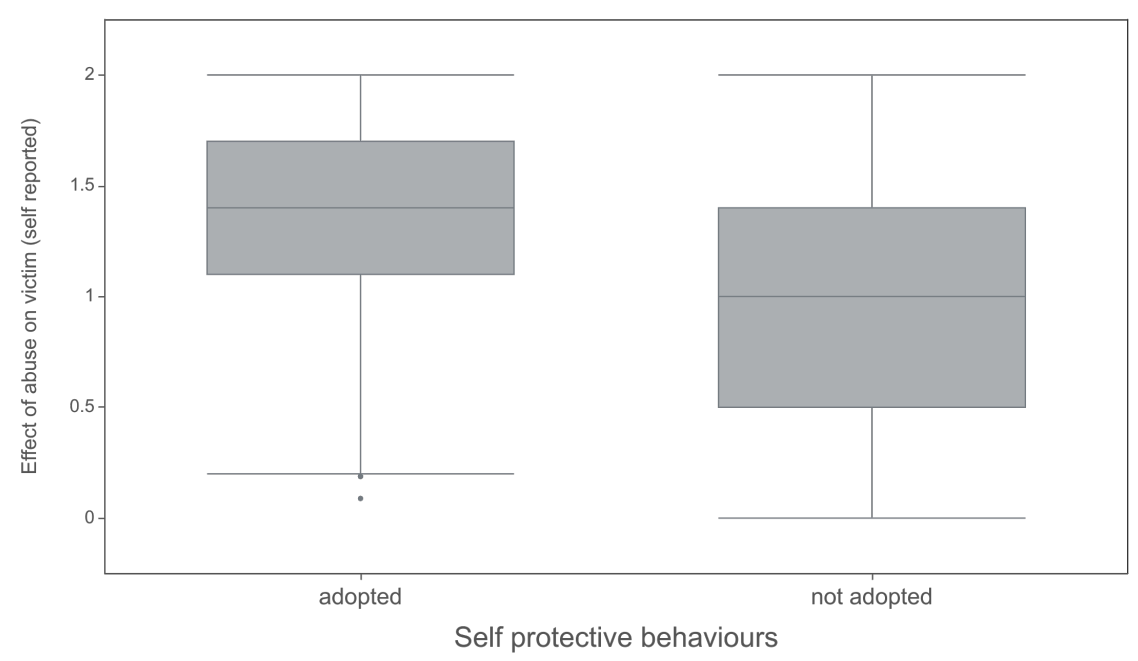

Figure 2: Boxplot of victim impact and adopting self-protective behaviours

Next, with the goal of measuring the combined effect of our explanatory variables on victim impact and adopting self-protective behaviour, and selecting the best explanatory model and to estimate the relative importance of our explanatory variables, we conducted two Bayesian variable selection analyses.

\section{Victim impact from cyber abuse}

Table 3 presents the results of the Bayesian variable selection analysis of the importance of coefficients included in the model explaining the level of perceived impact from different types of cyber abuse. While mixed cyber abuse has a relatively high inclusion probability of around $35 \%$, it is still well below the $50 \%$ mark, so it is not likely to contribute meaningfully to our explanatory model. Also, as Table 3 shows, by adding Mixed abuse to the explanatory model (See Model 2), the posterior probability of the model is reduced 
by nearly half making it a much less likely to be the best explanatory model. These findings suggest that the methods of cyber abuse are not, in fact, useful at explaining the perceived victim impact and instead it is the victim's gender and pre-existing offendervictim relationship that are the best explainants. Females with a pre-existing relationship with the offender are more likely to report a higher level of impact from cyber abuse victimization.

Table 3: Coefficients and models explaining the perceived level of impact from cyber abuse victimization

\begin{tabular}{|c|c|c|c|c|c|c|c|c|c|c|}
\hline \multirow[t]{2}{*}{ Coefficient } & \multirow[b]{2}{*}{ Mean } & \multirow[b]{2}{*}{$\mathrm{SD}$} & \multirow{2}{*}{$\begin{array}{r}2.5 \% \\
\text { HPD }^{* *}\end{array}$} & \multirow{2}{*}{$\begin{array}{r}97.5 \% \\
\mathrm{HPD}^{* *}\end{array}$} & \multirow{2}{*}{$\begin{array}{l}\text { Probability } \\
\text { of inclusion }\end{array}$} & \multicolumn{5}{|c|}{ Model* } \\
\hline & & & & & & 1 & 2 & 3 & 4 & 5 \\
\hline Constant & 0.903 & 0.069 & 0.775 & 1.051 & 100 & 0.917 & 0.867 & 1.044 & 0.906 & 0.869 \\
\hline Female & 0.168 & 0.046 & 0.077 & 0.254 & 99 & 0.167 & 0.171 & 0.172 & 0.167 & 0.174 \\
\hline Prior OVR & 0.163 & 0.071 & 0.000 & 0.263 & 92 & 0.163 & 0.179 & 0.000 & 0.173 & 0.180 \\
\hline Mixed abuse & 0.036 & 0.055 & 0.000 & 0.152 & 35 & - & 0.101 & - & - & - \\
\hline Indirect abuse & 0.001 & 0.015 & 0.000 & 0.000 & 5 & - & - & - & - & - \\
\hline $\operatorname{Age}^{* * *}$ & -0.001 & 0.005 & -0.010 & 0.000 & 5 & - & - & - & -0.011 & - \\
\hline Employed & 0.002 & 0.014 & 0.000 & 0.000 & 5 & - & - & - & - & 0.036 \\
\hline White & -0.001 & 0.011 & -0.021 & 0.000 & 4 & - & - & - & - & - \\
\hline Posterior probability (\%) & & & & & & 47 & 25 & 5 & 2 & 2 \\
\hline
\end{tabular}

$*$ Best 5 models (cumulative posterior probability $=81 \%$ )

** Highest Posterior Density

*** Standardized

In terms of the victim's gender and offender-victim relationship, they are included in all five best models with a cumulative posterior probability of over $80 \%^{13}$. As Fig. 3 shows, female victims are more likely than male victims to report higher impact from victimization. It is not clear why this might be the case, but we hypothesize that this effect may be related to the seriousness of abuse female victims experience compared with male victims. Also, it chimes with earlier research that suggests that female victims of conventional crime are more affected than male victims.

\footnotetext{
${ }^{13}$ The rest of possible models are not very likely
} 


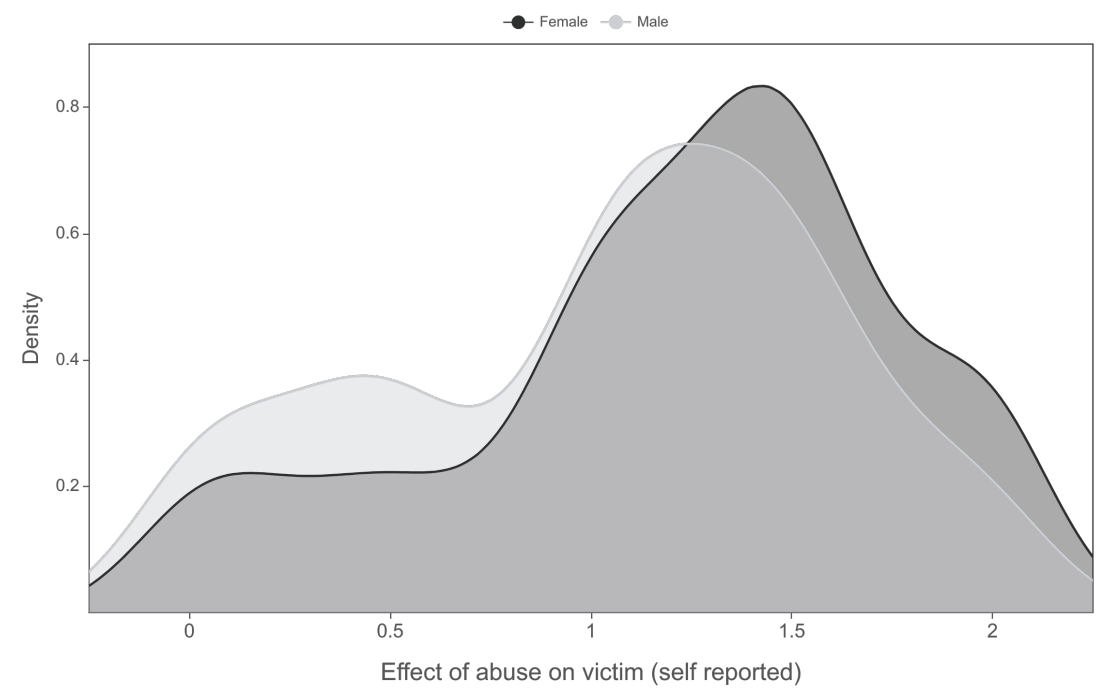

Figure 3: Density plot of victim impact vs. gender. Here, the darker grey density denotes female victims; light grey density - male victims

Females are more likely to report being abused by someone they knew prior to the attack, while males are more likely to be abused by a stranger. This seems to indicate that the nature of the triggers of interpersonal conflict may affect the perceived impact from cyber abuse. Research currently in progress will unpack the gendered nature of these triggers.

\section{Adopting self-protective behaviours}

The posterior means and standard deviations for each coefficient considered for explaining adopting self-protective behaviours following cyber abuse victimization are presented in Table 4. Using our data, perceived victim impact, age of the victim, prior offendervictim relationship and whether the victim experienced, mixed, indirect of direct type of cyber abuse are the factors that best explain the adoption of self-protective behaviours. All five best models include Impact, Abuse Methods, and Age and only 3 out of 5 best models include Gender. Variables Race, Employment and Motivation are not included in any of the best five models, meaning these variables do not explain adopting selfprotective behaviours following victimization. 
Table 4: Coefficients and models explaining adopting self-protective behaviours following cyber abuse victimization

\begin{tabular}{|c|c|c|c|c|c|c|c|c|c|c|}
\hline \multirow[t]{2}{*}{ Coefficient } & \multirow[b]{2}{*}{ Mean } & \multirow[b]{2}{*}{$\mathrm{SD}$} & \multirow{2}{*}{$\begin{array}{r}2.5 \% \\
\mathrm{HPD}^{* *}\end{array}$} & \multirow{2}{*}{$\begin{array}{r}97.5 \% \\
\mathrm{HPD}^{* *}\end{array}$} & \multirow{2}{*}{$\begin{array}{l}\text { Probability } \\
\text { of inclusion }\end{array}$} & \multicolumn{5}{|c|}{ Model* ${ }^{*}$} \\
\hline & & & & & & 1 & 2 & 3 & 4 & 5 \\
\hline Constant & 0.110 & 0.071 & -0.028 & 0.246 & 100 & 0.099 & 0.133 & 0.173 & 0.079 & 0.006 \\
\hline Impact & 0.299 & 0.033 & 0.235 & 0.362 & 100 & 0.300 & 0.304 & 0.311 & 0.287 & 0.287 \\
\hline Age $^{* * *}$ & 0.072 & 0.021 & 0.035 & 0.113 & 99 & 0.073 & 0.073 & -0.136 & 0.071 & 0.082 \\
\hline Prior OVR & -0.108 & 0.066 & -0.202 & 0.000 & 81 & -0.134 & -0.135 & 0.068 & -0.138 & - \\
\hline Mixed Abuse & 0.098 & 0.065 & 0.000 & 0.189 & 77 & 0.140 & 0.102 & - & 0.141 & 0.142 \\
\hline Indirect Abuse & -0.065 & 0.072 & -0.193 & 0.000 & 52 & - & -0.106 & -0.154 & - & - \\
\hline Female & 0.014 & 0.032 & 0.000 & 0.098 & 21 & - & - & - & 0.072 & - \\
\hline Employed & 0.001 & 0.011 & 0.000 & 0.000 & 5 & - & - & - & - & - \\
\hline White & -0.001 & 0.008 & 0.000 & 0.000 & 4 & - & - & - & - & - \\
\hline Posterior probability (\%) & & & & & & 23 & 14 & 12 & 8 & 5 \\
\hline
\end{tabular}

Based on the above, using our data we can conclude that experiencing higher impact from a cyber abuse victimization event is associated with a higher probability (57\%) of adopting self-protective behaviours. Older victims and those who experienced mixed abuse are more likely to adopt self-protective behaviours following an incident of victimization. In contrast, those who knew their abuser prior to the incident as opposed to those who were attacked by a stranger(s) are less likely to modify their behaviour (probability of adopting self-protective behaviours is 47\%). We might speculate that this is because where the victim is known to the offender, the victim recognizes that modifying technological behaviour alone will have a minimal effect-indeed, it may provoke the perpetrator to, for example, the shift from online to offline abuse.

Further, those who experienced indirect methods of cyber abuse are less likely to change their behaviour (probability of adopting self-protective behaviours is $48 \%$ ). This is likely to be explained through the nature of indirect abuse, which does not directly involve the victims, making adopting self-protective behaviours against an attack more or less futile. 


\section{Discussion and conclusion}

This study had a dual focus: to consider how the nature of the cyber abuse experienced and the characteristics of its victims affected both the perceived impact of the incident(s) and adoption of self-protective behaviours by victims. Consequently, our findings contribute to the larger literature on cyber abuse and impact from victimization.

While media often focuses on cyber abuse incidents on the extreme side of the spectrum of victim impact, the average impact in our sample was quite moderate (1.13 out of maximum 2). In line with the existing literature on victim impact from traditional and new technology-facilitated types of crimes, female victims reported experiencing higher perceived impact compared with male victims. Also, as expected from previous research, we found that those who had a prior relationship with their offender and women were more likely to say that they had been more affected by the abuse. Perhaps surprisingly, the type of cyber abuse experienced (direct, indirect or mixed) had a minimal effect on the psychological impact victims experienced. However, while we did not detect the differences in victim impact between different types of cyber abuse, the type of experienced cyber abuse actually plays a much more important role in adopting self-protective behaviours.

Forty per cent of victims of cyber abuse in our sample reported adopting some form of self-protective behaviours following their victimization. This is not dissimilar to findings in Nobles et al. (2014) who analyzed the 2006 Supplemental Victimization Survey to the National Crime Victimization that found that $51.01 \%$ of cyberstalking victims adopted at least one type of self-protective behaviours. The difference could be explained by the differences in sample collection methodologies and/or differences in definitions of self-protective behaviours.

Those who reported being affected the most were significantly more likely to adopt self-protective measures. On the other hand, women, older victims and those with no prior relationship with the offender were more likely to adopt self-protective behaviours. In the case of older victims, this may be a by-product of the relationship between age and offender-victim relationship, but it might reflect older victims being better informed 
about protective strategies. Future research should unpack the mechanism by which the age of the victim determines the likelihood of adopting self-protective behaviours. The fact that those with a prior close relationship with their abuser were less likely to adopt self-protective behaviours maybe because where the offender knows them well; merely changing internet usage may be considered insufficient.

Interestingly those who experienced mixed cyber abuse were significantly more likely to adopt self-protective behaviours (mixed cyber abuse was included in the best explanatory model with a probability of inclusion of $77 \%$ ) but experiencing indirect cyber abuse actually makes the victims less likely to adopt self-protective behaviours (with inclusion probability of just over 50\%). This suggests that victims feel that they do not have a lot of control over the victimization event and adoption of self-protective behaviours is not going to make any difference. The significant increase in chances of adopting self-protective behaviours by the victims of mixed cyber abuse is likely attributable to the inclusion in mixed abuse direct. By definition, mixed abuse requires that at least two different methods of abuse are present in one incident - at least one of which must be of the direct kind. If someone is sending you derogatory messages via email - it is easy to see how adopting a self-protective behaviour in the form of blocking the offending address could stop or at least temporarily prevent further victimization. However, if an offender is not involving the victim and instead uses third parties or online public spaces to commit the abuse (as in the case of indirect abuse), there is not much the victim can do to protect him or herself from re-victimization. This finding is quite significant as it provides additional evidence for the utility of a more detailed classification of cyber abuse that considers the nature of methods of abuse.

Our findings should be interpreted in light of the limitations of this study. First, considering the non-probability nature of our sample, we cannot generalize the findings to our target population of interest. Future research should test the findings using a sample randomly selected from the general population of American adults. Second, the data analyzed in this study is based on self-reports of victims of cyber abuse and may suffer from a number of biases, recall issues and other issues common for this type of 
data. While potentially limited by the self-report nature, victimization survey is the best currently available method of data collection on perceived victim impact. Allowing our respondents to use a slider rather than to have a binary response option (as was done in previous research) for measuring the perceived impact allowed us to have a much more nuanced data. And finally, the cross-sectional nature of the design of this study means that we could not establish whether the adoption of self-protective behaviours actually influences the risk of re-victimization. Future research should potentially utilize an experimental or longitudinal design to examine the effectiveness of self-protective behaviours against cyber abuse re-victimization.

Notwithstanding these limitations, the study contributes to several areas of knowledge. In particular, our findings suggest that similar to research in conventional types of crimes like stalking, victim impact from cyber abuse is perceived as more significant by female victims and those who had a relationship with the offender prior to the abuse. We also found no significant differences in perceived victim impact between different types of cyber abuse (direct, indirect and mixed abuse). However, our model was able to identify the important role the type of cyber abuse victimization experienced by the victim plays in adopting self-protective behaviours. Specifically, we found that while victims of mixed abuse were significantly more likely to adopt self-protective behaviours, in contrast, the victims of indirect abuse were less likely to so that. This indicates that the nature of the abuse experienced by the victim affects his or her decision making processes following the victimization, that is in line with previous literature that suggests the effect of offence type on the victim impact. These findings should help form coupled with future in-depth research in self-protective behaviours victims of different types of cyber abuse adopt to protect themselves from re-victimization should provide additional insight for developing better crime prevention strategies against cyber abuse. 


\section{References}

Baker, R., Blumberg, S., Brick, J., Couper, M., Courtright, M., Dennis, J., .. Lavrakas, P. (2010). Research synthesis: AAPOR report on online panels. Public Opinion Quarterly, 74, 711-781.

Baum, K., Catalano, S., Rand, M., \& Rose, K. (2009). Stalking victimisation in the United States. Retrieved from https://victimsofcrime.org/docs/src/baum-kcatalano-s-rand-m-rose-k-2009.pdf?sfvrsn $=0$

Behrend, T., Sharek, D., Meade, A., \& Wiebe, E. (2011). The viability of crowdsourcing for survey research. Behavior Research Methods, 43, 800-813.

Berinsky, A., Huber, G., \& Lenz, G. (2012). Evaluating online labor markets for experimental research: Amazon.com's Mechanical Turk. Political Analysis, 20, 351368.

Buhi, E., Clayton, H., \& Surrency, H. (2009). Stalking victimization among college women and subsequent help-seeking behavior. 57, 419-425.

Buhrmester, M., Kwang, T., \& Gosling, S. (2011). Amazon's Mechanical Turk: A new source of inexpensive, yet high-quality data? Perspectives on Psychological Science, $6,3-5$.

Chang, L.-C. \& Krosnick, J. (2009). National surveys via RDD telephone interviewing versus the Internet: Comparing sample representativeness and response quality. Public Opinion Quarterly, 73, 641-678.

Cho, H., Seon, J., Han, J. B., Smirnova, D., \& Kwon, I. (2019). Gender differences in the relationship between the nature of intimate partner violence and the survivor's help-seeking. Violence Against Women, 1-18.

Davis, K. E., Coker, A. L., \& Sanderson, M. (2002). Physical and mental health effects of being stalked for men and women. Violence and Victims, 17, 429-443.

Dinisman, T. \& Moroz, A. (2017). Understanding victims of crime: The impact of crime and support needs. Retrieved from https: / / www. victimsupport.org.uk/sites / default/files/VS_Understanding $\backslash \% 20$ victims $\backslash \% 20$ of $\backslash \% 20$ crime_web.pdf 
Dreßing, H., Bailer, J., Anders, A., Wagner, H., \& Gallas, C. (2014). Cyberstalking in a large sample of social network users: Prevalence, characteristics, and impact upon victims. Cyberpsychology, Behaviours and Social Networking, 17, 61-67.

Ellison, L. \& Akdeniz, Y. (1998). Cyber-stalking: The regulation of harassment on the internet (special edition: Crime, criminal justice and the internet). Criminal Law Review, 2948. Retrieved from http://www.cyber-rights.org/documents/stalking

Fisher, B., F.T., C., \& M.G., T. (2000). The sexual victimization of college women. Washington, DC: US Department of Justice.

Fisher, B., Cullen, F., \& Turner, M. (2002). Being pursued: Stalking victimization in a national study of college women. Criminology \& Public Policy, 1, 257-308.

Fissel, E. R. \& Reyns, B. W. (2019). The aftermath of cyberstalking: School, work, social, and health costs of victimization. American Journal of Criminal Justice.

Fissel, E. (2018). The reporting and help-seeking behaviours of cyberstalking victims. Journal of Interpersonal Violence, 1-26.

Gottfredson, M. R. \& Hindelang, M. J. (1979). A study of behaviour of law. American Sociaological Review, 44, 3-18.

Gramlich, J. (2019). 5 facts about crime in the u.s. Retrieved from https://www . pewresearch.org/fact-tank/2019/01/03/5-facts-about-crime-in-the-u-s/

Hensler-McGinnis, N. F. (2008). Cyberstalking victimization: Impact and coping responses in a national university sample. Dissertation.

Holmstrom, L. L. \& Burgess, A. W. (1978). The victim of rape: International reaction. Wiley; New York.

Johnson, M. C. \& Kercher, G. A. (2009). Identifying predictors of negative psychological reactions to stalking victimization. Journal of Interpersonal Violence, 24, 866-82. doi:10.1177/088626050831719

Korkodeilou, J. (2017). "No place to hide": Stalking victimisation and its psycho-social effects. International Review of Victimology, 23, 17-32. 
Kreuter, F., Presser, S., \& Tourangeau, R. (2008). Social desirability bias in CATI, IVR, and Web surveys: The effects of mode and question sensitivity. Public Opinion Quarterly, 72, 847-865.

Levay, K. E., Freese, J., \& Druckman, J. (2016). The demographic and political composition of mechanical turk samples. SAGE Open, 1, 1-17.

Marin, J.-M. \& Robert, C. (2007). Bayesian core: A practical approach to computational bayesian statistics. Springer.

Mawby, R. I. (2001). Burglary. Willan Publishing: Cullompton.

Mawby, R. I. \& Walklate, S. (1994). Critical victimology: International perspectives. Sage: London.

Mawby, R. \& Walklate, S. (1997). The impact of burglary: A tale of two cities. International Review of Victimology, 4, 267-295.

Nobles, M., Reyns, B., Fox, K., \& Fisher, B. (2014). Protection against pursuit: A conceptual and empirical comparison of cyberstalking an stalking victimization among a national sample. Justice Quarterly, 31, 986-1014.

Parsons-Pollard, N. \& Moriarty, L. J. (2009). Cyberstalking: Utilizing what we do know. Victims $\&$ Offenders, 4(4), 435-441.

Peer, E., Vosgerau, J., \& Acquisti, A. (2013). Reputation as a sufficient condition for data quality on Amazon Mechanical Turk. Behavior Research Methods, 46, 10231031.

Pickett, J., Roche, S., \& Pogarsky, G. (2018). Toward a bifurcated theory of emotional deterrence. Criminology.

Raftery, A. (1995). Bayesian model selection in social research. Sociological Methodology, 25, 111-163.

Reinhart, R. J. (2018). One in four americans have experienced cybercrime. Retrieved from https: / / news.gallup.com / poll / 245336 / one-four- americans- experiencedcybercrime.aspx 
Reyns, B., Henson, B., \& Fisher, B. (2011). A situational crime prevention approach to cyberstalking victimization: Preventive tactics for internet users and online place managers. Crime Prevention and Community Safety, 12, 99-118.

Shapland, J. \& Hall, M. (2007). What do we know about the effects of crime on victims? International Review of Victimology, 14, 175-217.

Sheridan, L. \& Lyndon, A. E. (2012). The influence of prior relationship, gender, and fear on the consequences of stalking victimization. Sex Roles: A Journal of Research, 66, 340-350. doi:http://dx.doi.org/10.1007/s11199-010-9889-9

Sheridan, L. \& Grant, T. (2007). Is cyberstalking different? Psychology, Crime $\&$ Law, 13.

Sherman, L., Neyroud, P. W., \& Neyroud, E. (2016). The Cambridge Crime Harm Index: Measuring total harm from crime based on sentencing guidelines. Policing, 1-13. doi:doi:10.1093/police/paw003

Short, E., Linford, S., Wheatford, J., \& Maple, C. (2014). The impact of stalking: The lived experience - a thematic analysis. Studies in Health Technology and Informatics, 199, 133-137.

Short, E., Guppy, A., Hart, J., \& Barnes, J. (2015). The impact of cyberstalking. Studies in Media and Communications, 3, 1-15.

Sue, V. \& Ritter, L. (2012). Conducting online surveys. Thousand Oaks, CA: Sage.

Tjaden, P. \& Thoennes, N. (2000). Prevalence and consequences of male-to-female and female-to-male intimate partner violence as measured by the national violence against women survey. Violence Against Women, 6, 241-161.

Tjaden, P. (1998). Stalking policies and research in the united states: A twenty year retrospective. European Journal on Criminal Policy Research, 15.

Tokunaga, R. S. \& Aune, K. S. (2017). Cyber-defense: A taxonomy of tactics for managing cyberstalking. Journal of Interpersonal Violence, 32(10), 1451-1475.

Vakhitova, Z. I., Webster, J. L., Alston-Knox, C. L., Reynald, D. M., \& Towsnley, M. K. (2018). Offender-victim relationship and offender motivation in the context of in- 
direct cyber abuse: A mixed-method exploratory analysis. International Review of Victimology, 24(3), 347-366. doi:10.1177/0269758017743073

Vakhitova, Z. \& Alston-Knox, C. (2018). Non-significant p-values? strategies to understand and better determine the importance of effects and interactions in logistic regression. PLoS ONE, 13(11). doi:https://doi.org/10.1371/journal.pone.0205076

Vakhitova, Z., Reynald, D., \& Townsley, M. (2016). Toward adapting routine activity and lifestyle exposure theories to account for cyber abuse victimization. Journal of Contemporary Criminal Justice, 32(2), 169-188.

Victim Support Agency. (n.d.). The effects of crime. Retrieved from https://www . victimsofcrime.vic.gov.au/the-crime/the-effects-of-crime

Weinberg, J. D., Freese, J., \& McElhattan, D. (2014). Comparing data characteristics and results of an online factorial survey between a population-based and a crowdsourcerecruited sample. Sociological Science, 1, 292-310.

Wilcox, P., Jordan, C., \& Pritchard, A. (2007). A multidimensional examination of campus safety: Victimization, perceptions of danger, worry about crime, and precautionary behavior among college women in the post-clery era. Crime $\mathcal{E}$ Delinquency, 53, 219-254.

Worsley, J., Wheatcroft, J., Short, E., \& Corcoran, R. (2016). Victim's voices: Understanding the emotional impact of cyberstalking and individual's coping responses. SAGE Open, 7, 1-13.

Ybarra, M., Price-Feeney, M., Lenhart, A., \& Zickuhr, K. (n.d.). Intimate partner digital abuse. Center for Innovative Public Health Research. Retrieved from https:// datasociety.net/pubs/oh/Intimate_Partner_Digital_Abuse_2017.pdf

Zellner, A. (1986). On assessing prior distributions and Bayesian regression analysis with g-prior distributions. In Bayesian inference and decision techniques: Essays in honor of Bruno de Finetti (Chap. 15, pp. 233-243). North-Holland ; Sole distributors for the U.S.A. and Canada, Elsevier Science Pub. Co. Retrieved from http://www.worldcat.org/isbn/0444877126 